\title{
Clinical Implication of Enlarged Prostate in Patients with the lleal Pouch-anal Anastomosis for Inflammatory Bowel Disease
}

\author{
Lei Lian, b, Emmanuel Obusez ${ }^{\mathrm{c}}$, Erick M. Remerc, Manoj Monga ${ }^{\mathrm{d}}$, \\ Bo Shen ${ }^{\mathrm{a}, \mathrm{e}}$
}

\begin{abstract}
Background: Enlarged prostate is often noticed in patients with ileal pouch-anal anastomosis (IPAA) in our clinical practice. The aims were to identify the factors associated with enlarged prostate and to investigate its clinical implications.

Methods: IPAA patients with available prostate imaging after IPAA were included. Prostate length and width were measured in the axial plane and height in coronal plane. Prostate volume was calculated with the formula (length $\times$ width $\times$ height $) \times \pi / 6$. A volume greater than $40 \mathrm{~cm}^{3}$ was used to define enlarged prostate.

Results: Prostate enlargement was found in 58 (24.8\%) out of 234 patients. Factors associated with prostate enlargement included advanced age at imaging examination $(55.6 \pm 11.5$ vs. $41.3 \pm 13.6$ years, $\mathrm{P}<0.0001)$, age at pouch surgery $(46.0 \pm 11.8$ vs. $32.5 \pm 12.9$ years, $\mathrm{P}<0.0001)$, and the presence of an S-pouch $(6.9 \%$ vs. $1.1 \%, \mathrm{P}=0.03)$. Postoperative use of biologics was less common in patients with enlarged prostate $(5.2 \%$ vs. $17 \%, \mathrm{P}=0.03)$. However, pouch duration was comparable $(10.0 \pm 5.9$ vs. $8.8 \pm 6.8$ years, $\mathrm{P}=0.2)$ and pouch failure rate was similar. A trend towards an increased risk for acute pouchitis in patients with enlarged prostate was noticed (19\% vs. $9.1 \%, \mathrm{P}=0.06$ ). The association of S-pouch (odds ratio: $7.2,95 \%$ confidence interval: 1.1 - 46.4) and enlarged prostate remained significant after adjusting for age, acute pouchitis, and redo pouch on multivariate analysis.
\end{abstract}

Conclusions: Prostate enlargement appears to be uncommon after

Manuscript submitted January 26, 2018, accepted January 31, 2018

aDepartment of Gastroenterology and Hepatology, The Cleveland Clinic Foundation, Cleveland, OH 44195, USA

bDepartment of Colorectal Surgery, The Cleveland Clinic Foundation, Cleveland, OH 44195, USA

'Department of Diagnostic Radiology, The Cleveland Clinic Foundation, Cleveland, OH 44195, USA

dDepartment of Urology, The Cleveland Clinic Foundation, Cleveland, $\mathrm{OH}$ 44195, USA

${ }^{\mathrm{e} C o r r e s p o n d i n g ~ A u t h o r: ~ B o ~ S h e n, ~ D i g e s t i v e ~ D i s e a s e ~ a n d ~ S u r g e r y ~ I n s t i t u t e-~}$ A31, The Cleveland Clinic Foundation, 9500 Euclid Ave, Cleveland, $\mathrm{OH}$ 44195, USA. Email: shenb@ccf.org

doi: https://doi.org/10.14740/gr975e
IPAA and it was associated with S-pouch configuration and advanced age. Enlarged prostate in the setting of IPAA does not seem to have adverse impact on pouch outcomes, although there is a trend in correlation between enlarged prostate and acute pouchitis.

Keywords: Ileal pouch-anal anastomosis; Prostate; Restorative proctocolectomy

\section{Introduction}

Ulcerative colitis (UC) is one of the two most common forms of inflammatory bowel disease (IBD) worldwide with a progressively increasing incidence [1]. More than $20 \%$ of patients with UC will eventually require surgical management [2], for which the procedure of choice is total proctocolectomy (TPC) with ileal-pouch anal anastomosis (IPAA). IPAA obviates the need for a permanent end ileostomy, preserves the route of natural defecation, and maintains fecal continence. This surgical procedure has been demonstrated to have a high success rate of up to $90-95 \%$ with improved functional outcome and quality of life (QOL) [3].

However, IPAA has been shown to be associated with adverse events including pouchitis, anastomotic leak, anal stricture, pelvic sepsis, and small bowel obstruction [4], which may result in pouch failure with pouch excision or permanent diversion in $3.5-10 \%$ of cases $[5,6]$. Approximately half of the male populations electing to undergo IPAA are male with an average age of $40-50$ years $[7,8]$. The resection of colon and rectum leaves the pelvic space to be filled with the fashioned ileal pouch, which is in the close proximity to the prostate gland. A healthy or diseased ileal pouch and prostate may have an impact on either organ's functional outcome. The proximity makes local management of prostate cancer in male IPAA patients challenging [9].

In our clinical practice, we have noticed that an enlarged prostate was common in patients with IPAA. Its risk factors and impact on pouch outcomes are not known. We hypothesized that the presence of an ileal pouch may lead to prostate enlargement due to the close proximity between the two organs and these morphologic changes may be associated with pouch inflammation. The aims of the study were to identify the factors associated with enlarged prostate and to investigate its 
clinical implications.

\section{Patients and Methods}

\section{Patients}

The study was approved by the Institutional Review Board (IRB) at the Cleveland Clinic Foundation. All eligible male patients with IPAA were identified from a prospectively maintained pouchitis database. Prostate imaging (either computerized tomography or magnetic resonance) were procured through electronic query and field search. Images were reviewed and analyzed on an Impax 6.0 PACS (AGFA healthcare, Siemens, Mortsel, Belgium).

\section{Inclusion criteria and exclusion criteria}

Men with IPAA who had available imaging for prostate gland volume measurement were included in this study. Patients with a preoperative diagnosis of UC, indeterminate colitis (IC), or Crohn's colitis were included. Patients with Kock pouch or familial adenomatous polyposis were excluded.

\section{Study variables}

Patient demographics, smoking, family history of cancer (prostate cancer, colon cancer, etc.) and IBD-related characteristics (disease duration, diagnosis at the time of colectomy (UC vs. Crohn's colitis vs. indeterminate colitis), indication for colectomy, extent of colitis, and primary sclerosing cholangitis (PSC), and pouch-related variables (configuration of pouch, number of stage of pouch surgery, duration of pouch, pouch disease diagnosis, and current use of anti-tumor necrosis factor biologics/immunomodulators) were obtained from the database. Patient electronic medical records were reviewed to collect data regarding prostate gland related features (history of prostatic diseases, prostate specific antigen (PSA), prostate volume before and after IPAA). We looked to see if there were any concurrent autoimmune diseases including asthma, diabetes mellitus, rheumatoid arthritis, thyroid disease, psoriasis, Sjogren syndrome, Raynaud's phenomenon, systemic lupus erythema, hemolytic/pernicious anemia, vitiligo, idiopathic thromboembolic purpura, celiac disease, multiple sclerosis, autoimmune pancreatitis, and autoimmune hepatitis.

\section{Outcome measurements}

The length and width of the prostate were measured in the axial plane and height from the coronal plane. (Fig. 1) The prostate gland volume was calculated using the formula (length $\times$ width $\times$ height $) \times \pi / 6[10,11]$. Prostate size varies with age. We set up prostate glands with a volume greater than $40 \mathrm{~cm}^{3}$ as an enlarged prostate gland in this study. The association of the enlarged prostate and clinical variables was assessed.

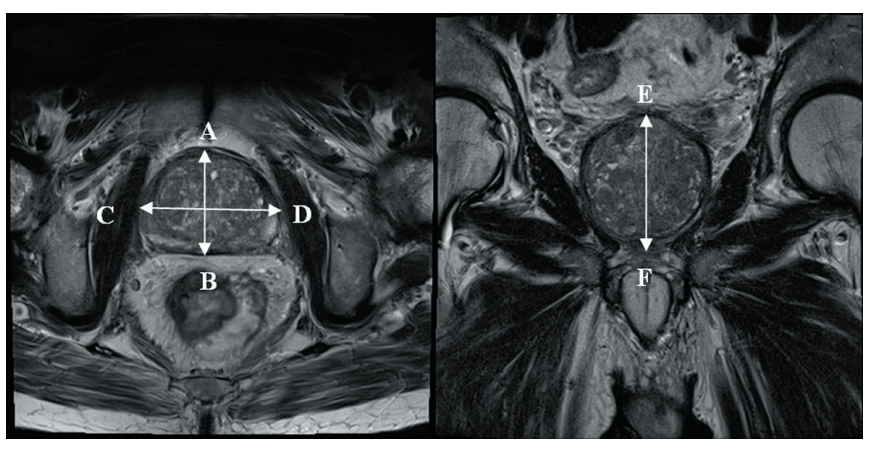

Figure 1. Axial (left) and coronal (right) MRI images showing the methodology for measurement of prostate volume: anterior-posterior diameter (A-B) of $46 \mathrm{~mm}$, width (transverse) (C-D) of $62 \mathrm{~mm}$, and height (cranio-caudal) (E-F) of $62 \mathrm{~mm}$.

\section{Statistical analysis}

The association of the enlarged prostate with patient demographics, underlying disease, characteristics of IPAA, and pouch diagnosis were analyzed. Descriptive analyses were used to summarize the data procured. These included mean/ standard deviation for continuous factors, and frequency/percentage for categorical factors. Univariate analysis (Chi-square or Fisher's exact tests) was used for categorical variables and Student's $t$-tests or Wilcoxon rank sum tests for continuous variables. Differences will be deemed statistically significant with a $P$ value less than 0.05 .

\section{Results}

A total of 234 male patients with IPAA and available post-IPAA prostate gland imaging were included in this study. Ethnic demographics included 221 (94.4\%) Caucasian patients, five (2.1\%) African American patients, and eight (3.4\%) patients of "other" ethnicity. The mean age was $44.9 \pm 14.5$ years at the time of prostate measurement and $35.8 \pm 13.9$ years at the time of pouch construction. The mean duration of UC diagnosis to pouch creation was $9.6 \pm 7.6$ years. Indications for colectomy varied among the patients with refractory UC in $202(86.3 \%)$ and development of dysplasia in 32 (13.7\%). Pouch surgery was 1-stage IPAA in two $(0.9 \%)$ patients, 2 -stage IPAA in 122 $(52.1 \%)$, 3-stage in $70(29.9 \%)$, and the remaining $40(17.1 \%)$ patients had a redo pouch. Since disease status of ileal pouch is not static, we used the assessment of last pouch center visit as the final diagnosis. The final pouch diagnosis in these patients were as follows: normal pouch in $13(5.6 \%)$ patients, irritable pouch syndrome (IPS) in 30 (12.8\%), active pouchitis in 27 $(11.5 \%)$, refractory pouchitis in $40(17.1 \%)$, Crohn's disease (CD) of the pouch in $59(25.2 \%)$, cuffitis in $22(9.4 \%)$, and surgical complications of the pouch in $43(18.4 \%)$.

\section{Prostate gland volume measurement}

The mean length of prostate gland in this cohort was $3.6 \pm$ 
Table 1. Association of Enlarged Prostate and General Clinical Variables

\begin{tabular}{llll}
\hline Variable & Enlarged $(\mathbf{n}=\mathbf{5 8})$ & Normal $(\mathbf{n}=\mathbf{1 7 6})$ & P value \\
\hline Age at prostate volume measurement & $55.6 \pm 11.5$ & $41.3 \pm 13.6$ & $<0.0001$ \\
Age at ulcerative colitis diagnosis & $33.9 \pm 12.4$ & $23.7 \pm 11.7$ & $<0.0001$ \\
Smoker & $14(24.1 \%)$ & $32(18.2 \%)$ & 0.34 \\
Chronic non-steroidal anti-inflammatory drug use & $4(6.9 \%)$ & $11(6.2 \%)$ & 0.99 \\
Concurrent autoimmune disease & $7(12.1 \%)$ & $25(14.2 \%)$ & 0.83 \\
\hline
\end{tabular}

$0.8 \mathrm{~cm}$, the mean width $4.5 \pm 0.7 \mathrm{~cm}$, and the mean height $3.8 \pm 0.9 \mathrm{~cm}$. The mean prostate volume was calculated using the aforementioned formula at $33.9 \pm 19.1 \mathrm{~cm}^{3}$. Using a cutoff of $40 \mathrm{~cm}^{3}$, patients were classified as having an enlarged prostate gland $(\mathrm{n}=58)$ versus a normal prostate gland $(\mathrm{n}=$ 176).

\section{Association of prostate gland enlargement and clinical characteristics}

Patients with prostate gland enlargement were older at the time of measurement and at the diagnosis of ulcerative colitis. No statistically significant differences were found in smoking history, chronic use of nonsteroidal anti-inflammatory drugs (NSAID), and concurrent autoimmune diseases (Table 1). Univariate analysis showed no significant association of enlarged prostate and underlying IBD characteristics (Table 2) including family history of IBD, indication for colectomy, extent of IBD, toxic megacolon, or the use of biologics before colectomy. PSA level was tested in only 23 patients and there was no significant association between PSA level and prostate enlargement $(3.81 \pm 3.04$ vs. $1.5 \pm 1.33, \mathrm{P}=0.06)$.

\section{Association of enlarged prostate and pouch-related char- acteristics}

As detailed in Table 3, redo pouch was more common in patients with an enlarged prostate gland; however the difference was not statistically significant. Although age differed, the duration of pouch was comparable between patients with enlarged and normal prostate glands. The presence of an S-pouch was significantly associated with prostate gland enlargement, as was the postoperative use of biologics. The postoperative use of immunosuppressants was similar in patients with normal and enlarged prostate glands.

A trend towards increased acute pouchitis in patients with enlarged prostate was noticed, however the difference was not statistically significant ( $19 \%$ vs. $9.1 \%, \mathrm{P}=0.056)$. There was no significant association between prostate gland volume and pouch failure.

Table 2. Association of Enlarged Prostate and Underlying Inflammatory Bowel Disease Characteristics

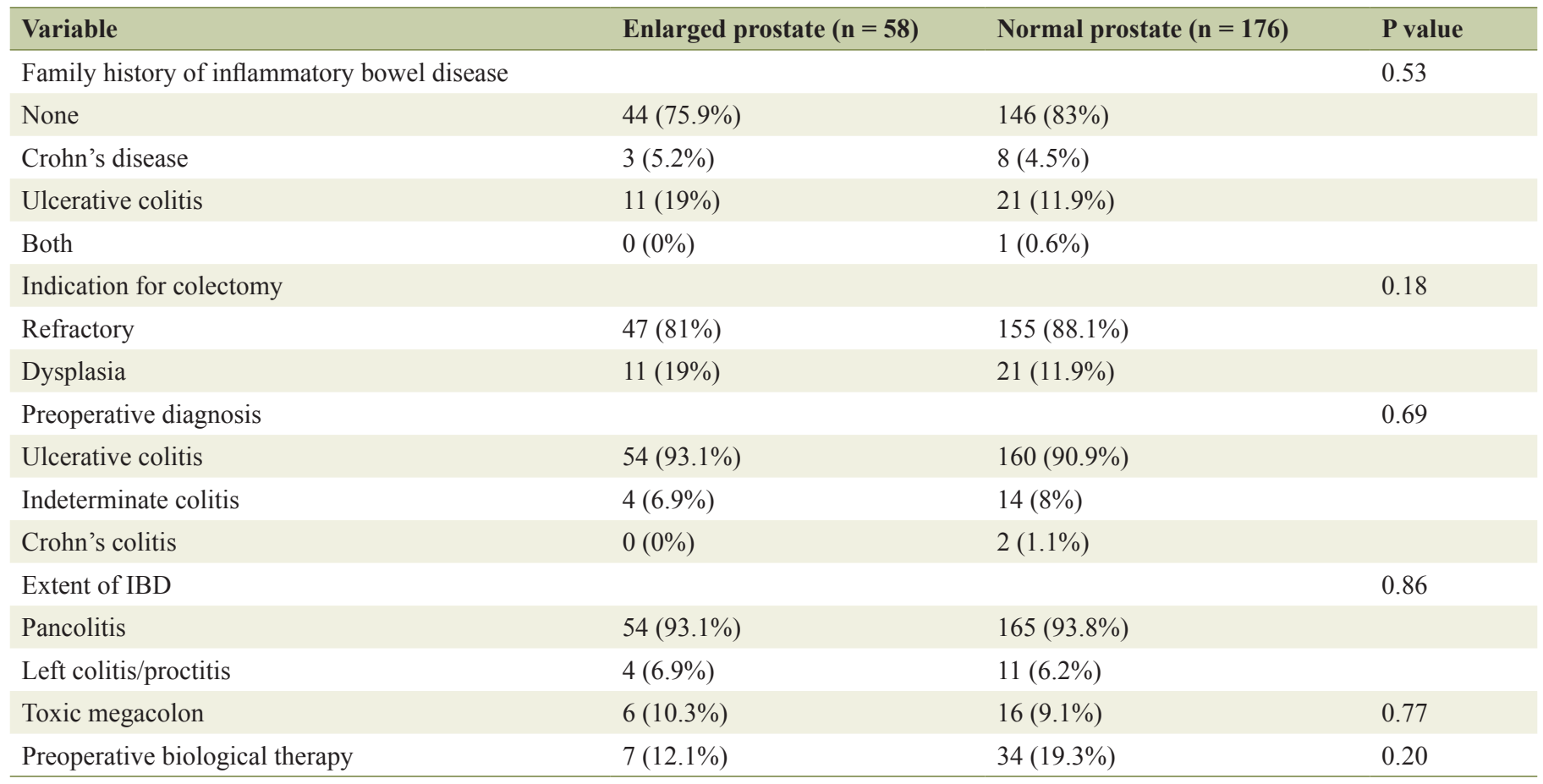


Table 3. Association of Enlarged Prostate and Pouch-related Features

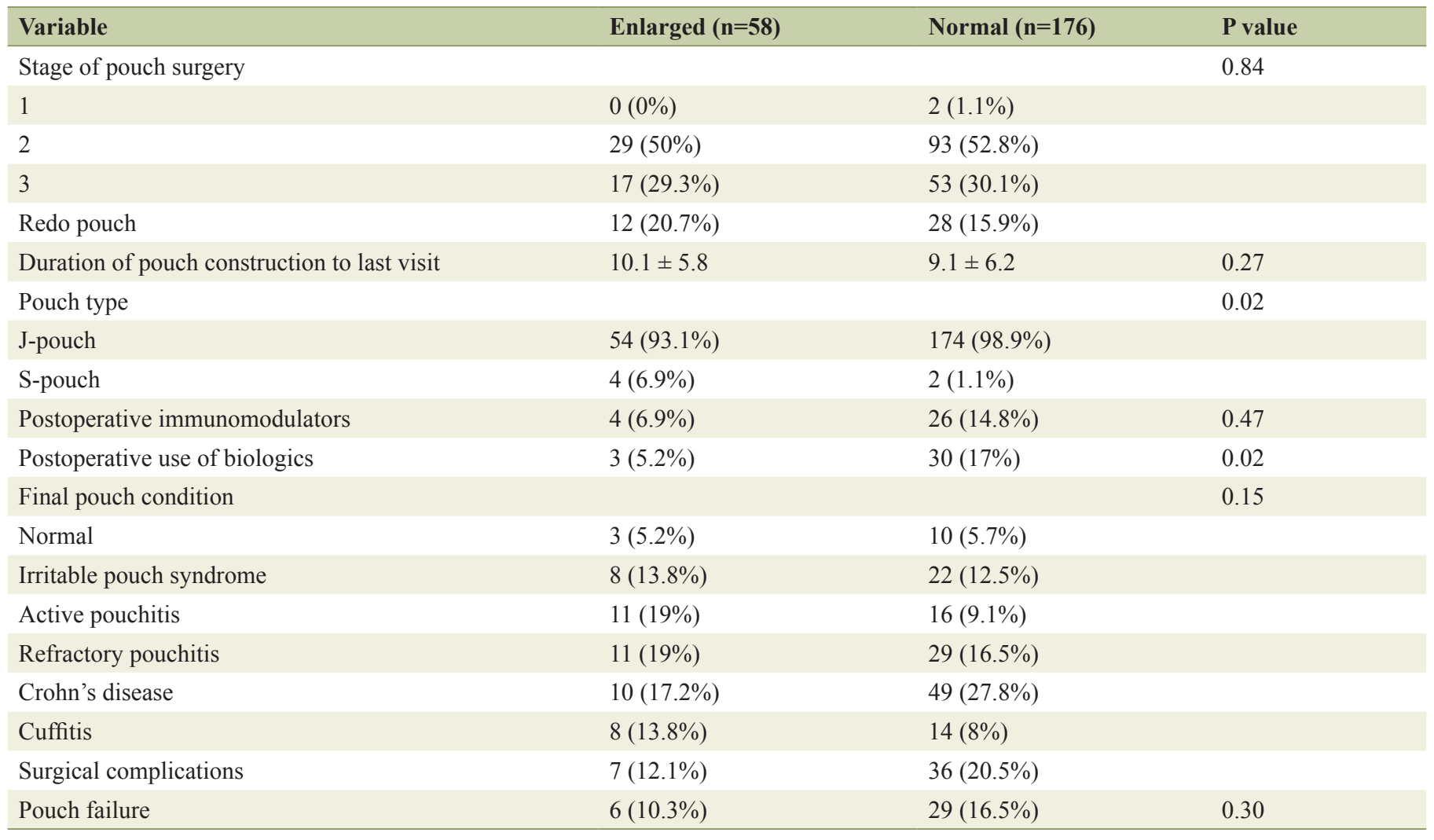

\section{Multivariate analysis of association between enlarged prostate and clinical variables}

A multivariate logistic regression model was performed to evaluate the association between an enlarged prostate gland and other clinical variables. As shown in Table 4, the presence of an S-pouch was associated with seven-fold increased risk of prostate gland enlargement, remaining statistically significant after adjusting for age and redo pouch on multivariate analysis.

\section{Discussion}

Our study found that enlarged prostate gland was common in patients with IPAA. Prostate gland enlargement was associated with both age and pouch configuration. However, pouch disease conditions, pouch failure, redo pouch surgery, and

Table 4. Multivariate Analysis of Risk Factors of Enlarged Prostate

\begin{tabular}{lll}
\hline Variable & Odds ratio $(\mathbf{9 5 \%}$ confidence interval) & P value \\
\hline Age & $1.1(1.05-1.11)$ & 0 \\
Acute pouchitis & $2.4(0.9-6.2)$ & 0.07 \\
S-pouch & $7.2(1.1-46.4)$ & 0.04 \\
Redo pouch & $1.4(0.6-3.4)$ & 0.42 \\
\hline
\end{tabular}

smoking history were not found to be associated with an enlarged prostate. To the best of our knowledge, there have been no prior studies examining the association of these variables.

The surgical procedures TPC and IPAA involve both colonic and rectal dissection. Throughout the latter part of both surgeries, the rectum is dissected anteriorly to the prostate in male patients. Subsequently an ileal pouch is placed in the immediate vicinity of the prostate gland. The previous studies on interaction between an ileoanal pouch and the prostate gland were limited only to treatment of prostate cancer in pouch patients. In a retrospective review, Umbreit et al [12] included 16 patients undergoing a prostatectomy after IPAA and found that prostatectomy was successfully achieved in all patients without pouch violation or pouch-related postoperative complications. The authors also found overall pouch function remained unchanged after prostatectomy. Another study by Williamson et al [13] involving brachytherapy for localized prostate cancer in patients with IPAA showed no serious pouch complications, such as pouch ulcers, fistulas, or fecal incontinence [13]. In these patients, bowel frequency worsened immediately after brachytherapy. However, all patients returned to their baseline bowel pattern 4 months after the procedure [13].

Our study focuses on changes in prostate volume after IPAA. One of the strongest correlations was found between advanced age and prostate volume. The average age of the enlarged prostate group was approximately 10 years older than the normal prostate group. This association characterized between age and prostate volume is not surprising. There have 
been multiple studies relating increasing prostate volume to age $[14,15]$ which had been ascribed to hormonal levels [16, 17].

The present study showed an association between an Spouch configuration and enlarged prostate. An S-pouch is constructed using three limbs of 12 to $15 \mathrm{~cm}$ of terminal small bowel and may be usually created to excessive tension in the anastomosis [18]. A possible explanation for the S-pouch configuration and association with prostate enlargement may be the complex nature of the suturing involved in the S-pouch procedure as compared with simpler J-pouch configuration. The creation of the S-pouch is by hand-sewn anastomosis to the anal transitional zone, which has an increased manipulation of tissue adjacent to the prostate.

No increased risk for adverse outcomes was found to be associated with prostate enlargement in UC patients with an ileal pouch. Therefore, patient assurance should be given if an enlarged prostate is found on imaging studies and aggressive investigation may not be necessary.

Our study was limited by its retrospective study design. The dynamic changes in prostate volume cannot be assessed because constant monitoring of prostate size with imaging studies is not feasible. It would be more effective if we had access to multiple scans throughout a specific time span, leading to more reliable data. Additionally the enlarged prostate could be due to other confounding factors, such as age or hormonal level. A longitudinal study measuring the prostate volume before and after TPC and IPAA is warranted to further illustrate the impact of the surgery on the morphology of the prostate. Finally, the study had selection and referral bias due to the nature of our Pouch Center. We established the Center for Ileal Pouch Disorder at the Cleveland Clinic in 2002. Up to the date of the censor of the data, we had 1,520 patients in our Pouchitis Registry. A total of 435 patients had images of computed tomography (CT) or magnetic resonance imaging (MRI) which were available to review, among whom 234 patients were males. We included all 234 patients in the study. Due to the nature of the Center for Ileal Pouch Disorders, more than $90 \%$ of patients had various forms of ileal pouch disorders, ranging from irritable pouch syndrome to $\mathrm{CD}$ of the pouch. In fact, only 13 $(4.3 \%)$ patients had normal pouches. Transabdominal imaging such as CT and MRI have been routinely performed in patients with diseased pouches, ranging from chronic pouchitis to $C D$ of the pouch. Therefore, the studied patient population in this cohort may not reflect true general pouch population in which the majority of patients have normal functioned pouches. To verify our findings, a prospective study with cross-sectional imaging would be performed in all patients with healthy or diseased pouches.

In conclusion, prostate enlargement is common in patients with IPAA, which did not appear to be associated with a poor pouch outcome. Advanced age and pouch configuration were found to be factors associated with the enlarged prostate.

\section{Acknowledgments}

The study was partially supported by the Ed and Joey Story
Endowed Chair (to B.S.)

\section{Disclosure}

The authors declared no financial conflict of interest.

\section{Abbreviations}

CD: Crohn's disease; CT: computed tomography; EHR: electronic health record; IBD: inflammatory bowel disease; ICD: the International Classification of Disease; IPAA: ileal pouchanal anastomosis; IPS: irritable pouch syndrome; IRB: the Institutional Review Board; MRI: magnetic resonance imaging; NSAID: non-steroidal anti-inflammatory drugs; PSA: prostate-specific antigen; PSC: primary sclerosing cholangitis; QOL: quality of life; TPC: total proctocolectomy; UC: ulcerative colitis

\section{Reference}

1. Danese S, Fiocchi C. Ulcerative colitis. N Engl J Med. 2011;365(18):1713-1725.

2. Farmer RG, Easley KA, Rankin GB. Clinical patterns, natural history, and progression of ulcerative colitis. A long-term follow-up of 1116 patients. Dig Dis Sci. 1993;38(6):1137-1146.

3. Fazio VW, Ziv Y, Church JM, Oakley JR, Lavery IC, Milsom JW, Schroeder TK. Ileal pouch-anal anastomoses complications and function in 1005 patients. Ann Surg. 1995;222(2):120-127.

4. Shen B, Remzi FH, Lavery IC, Lashner BA, Fazio VW. A proposed classification of ileal pouch disorders and associated complications after restorative proctocolectomy. Clin Gastroenterol Hepatol. 2008;6(2):145-158; quiz 124.

5. Belliveau P, Trudel J, Vasilevsky CA, Stein B, Gordon $\mathrm{PH}$. Ileoanal anastomosis with reservoirs: complications and long-term results. Can J Surg. 1999;42(5):345-352.

6. Meagher AP, Farouk R, Dozois RR, Kelly KA, Pemberton JH. J ileal pouch-anal anastomosis for chronic ulcerative colitis: complications and long-term outcome in 1310 patients. Br J Surg. 1998;85(6):800-803.

7. Fazio VW, Kiran RP, Remzi FH, Coffey JC, Heneghan HM, Kirat HT, Manilich E, et al. Ileal pouch anal anastomosis: analysis of outcome and quality of life in 3707 patients. Ann Surg. 2013;257(4):679-685.

8. Shen B, Fazio VW, Remzi FH, Delaney CP, Bennett AE, Achkar JP, Brzezinski A, et al. Comprehensive evaluation of inflammatory and noninflammatory sequelae of ileal pouch-anal anastomoses. Am J Gastroenterol. 2005;100(1):93-101.

9. Shen B, Angermeier KW, Remzi FH, Katz S. Screening and diagnosis of prostate cancer in patients with ileal pouch-anal anastomosis: consensus from an expert panel. Am J Gastroenterol. 2011;106(2):186-189. 
10. Aarnink RG, de la Rosette JJ, Debruyne FM, Wijkstra H. Formula-derived prostate volume determination. Eur Urol. 1996;29(4):399-402.

11. Jeong CW, Park HK, Hong SK, Byun SS, Lee HJ, Lee $\mathrm{SE}$. Comparison of prostate volume measured by transrectal ultrasonography and MRI with the actual prostate volume measured after radical prostatectomy. Urol Int. 2008;81(2):179-185.

12. Umbreit EC, Dozois EJ, Crispen PL, Tollefson MK, Karnes RJ, Blute ML. Radical prostatectomy for prostate cancer after ileal pouch-anal anastomosis offers oncologic control and sustains quality of life. J Am Coll Surg. 2010;210(2):232-239.

13. Williamson R, Smaldone MC, Gibbons EP, Smith RP, Beriwal S, Benoit RM. Prostate brachytherapy after ileal pouch-anal anastomosis reconstruction. Urology. 2009;73(2):369-373.

14. Berges R, Oelke M. Age-stratified normal values for prostate volume, PSA, maximum urinary flow rate, IPSS, and other LUTS/BPH indicators in the German male community-dwelling population aged 50 years or older. World J Urol. 2011;29(2):171-178.

15. Zhang SJ, Qian HN, Zhao Y, Sun K, Wang HQ, Liang GQ, Li FH, et al. Relationship between age and prostate size. Asian J Androl. 2013;15(1):116-120.

16. Partin AW, Oesterling JE, Epstein JI, Horton R, Walsh PC. Influence of age and endocrine factors on the volume of benign prostatic hyperplasia. J Urol. 1991;145(2):405409.

17. Zeng QS, Xu CL, Liu ZY, Wang HQ, Yang B, Xu WD, Jin TL, et al. Relationship between serum sex hormones levels and degree of benign prostate hyperplasia in Chinese aging men. Asian J Androl. 2012;14(5):773-777.

18. Kirat HT, Remzi FH. Technical aspects of ileoanal pouch surgery in patients with ulcerative colitis. Clin Colon Rectal Surg. 2010;23(4):239-247. 